\title{
EXPLOITING SPARSITY IN REMOTE SENSING AND EARTH OBSERVATION: THEORY, APPLICATIONS AND FUTURE TRENDS
}

\author{
Xiao Xiang Zhu ${ }^{(1,2)}$, Richard Bamler ${ }^{(1,2)}$ \\ (1) Remote Sensing Technology Institute (IMF), German Aerospace Center (DLR), Germany \\ (2) Technische Universität München, Germany
}

Tutorial for Invited Session “Sparse Reconstruction and Compressive Sensing in Remote Sensing”

\begin{abstract}
Sparse signals are commonly expected in remote sensing and Earth observation. Along with the significant development of the compressive sensing theory, exploitation of sparsity in remote sensing became a very relevant and active field. Breakthroughs are brought in different remote sensing problems covering synthetic aperture radar, multispectral and hyperspectral image analysis, and LiDAR. Tailored to this special session, this tutorial gives a review, to the best knowledge of the session chair, on recent advances in sparsity exploitation in remote sensing and Earth observation, regarding the theory, applications and future trends.
\end{abstract}

Index Terms - sparsity exploitation, compressive sensing, remote sensing, synthetic aperture radar, hyperspectral imaging, optical remote sensing

\section{THEORY}

Prominent problems in remote sensing for earth observation are:

- Notoriously ill-conditioned and undetermined inverse problems

A typical example is super-resolution for radar, optical and hyperspectral imaging systems. In order to achieve good resolution and positioning accuracy the image to be reconstructed must be sampled much more densely than the resolution unit defined by the diffraction limit. This results in underdetermined systems.

- Non-Gaussian statistics and a large amount of outliers
Many remote sensing techniques suffer from unmodeled noise contributions (e.g. turbulent atmospheric and ionospheric delay) and a large amount of outliers (e.g. by multi-path effects). Typical examples are Lidar or persistent scatterer interferometry.

- Expensive sensors and high data rate Advanced applications call for sensors with higher and higher resolution which leads to high requirements on the involved hardware and software. E.g. for radar systems, high pulse repetition frequency is required to sample higher resolution data which will lead to expensive sensors and renders the data rate high, although the information content does not grow accordingly.

The concept of sparsity offers a solution to many of these problems.

Let $\mathbf{x}$ be the signal to be reconstructed with a length of $L$ and $\mathbf{y}$ be the measurement vector having $N$ elements. The remote sensing measurement acquisition can be generally modeled as:

$$
\mathbf{y}=\mathbf{F}(\mathbf{x})+\mathbf{\varepsilon}
$$

Where $\mathbf{F}(\cdot)$ is the - possibly nonlinear - forward model and $\boldsymbol{\varepsilon}$ is the measurement noise. Linearizing the underlying measurement model yields:

$$
\mathbf{y}=\mathbf{K x}+\boldsymbol{\varepsilon}
$$

Where $\mathbf{K}$ is the sensing matrix (i.e. the Jacobian of $\mathbf{F}$ ). Often the system model of eq. 2 is an underdetermined inverse problem, i.e. $N<L$, and appropriate regularization is required in order to obtain a robust 
estimate of $\mathbf{x}$. If $\mathbf{x}$ is sparse, i.e. compared to its length $L$ it has only few non-zero elements or its projection onto an orthogonal basis $\boldsymbol{\Phi}$ (e.g. Fourier, wavelet) has only few non-zero coefficients, this sparsity property can be used as a strong prior for regularizing the underdetermined inverse problem. Among the infinitely many solutions of the (noise-free) underdetermined system, the sparsest solution, i.e. the solution with $\boldsymbol{\Phi} \hat{\mathbf{x}}$ having the minimum $L_{0}$ norm, is assumed to be the most probable one. Since the measurements are contaminated with noise, the $L_{0}$ norm is jointly minimized with the classical residual term:

$$
\hat{\mathbf{x}}=\arg \min _{\mathbf{x}}\left\{\|\mathbf{y}-\mathbf{K x}\|_{2}^{2}+\lambda_{0}\|\mathbf{\Phi} \mathbf{x}\|_{0}\right\}
$$

where $\lambda_{0}$ is a regularization parameter balancing sparsity and residuals (noise). Eq.3 gives the theoretically most probable sparse solution. However, this optimization task is N-P hard, and hence is not applicable in practice. It can also give multiple and unrealistic high-energy solutions. A practical approach provided by compressive sensing (CS) is that under certain constraints [1], the $L_{0}$ norm prior used in eq. 1 can be well approximated by the $L_{1}$ norm. This leads to an optimization task that mixes $L_{2}$ and $L_{1}$ expressions in the form:

$$
\hat{\mathbf{x}}=\arg \min _{\mathbf{x}}\left\{\|\mathbf{y}-\mathbf{K x}\|_{2}^{2}+\lambda_{1}\|\mathbf{\Phi} \mathbf{x}\|_{1}\right\}
$$

This approximation renders the problem convex and solvable by linear programming.

Besides this $L_{2}$ and $L_{1}$ norm minimization several other minimizers are proposed in sparse signal representation, e.g. for robust PCA computation [2], exploiting joint sparsity [3], etc.

\section{APPLICATIONS}

In the recent years, pioneer research has been carried out to apply this model for solving remote sensing problems that lead to exciting results. Selected remote sensing problems addressed by the community are as follows.

- SAR imaging [4]-[6]: Modern SAR sensors provide very high spatial resolution. This high resolution reduces the information content per pixel, and hence renders the signal medium sparse in azimuth and range. This is particularly true for sparse scenes, e.g. in coastal areas [7].

- Optimizing remote sensing systems [8]: Sparse signals can be well reconstructed from much fewer samples than the Shannon sampling theory requires. A straightforward application is to use the compressive sensing paradigm to design innovative imaging system aiming at acquiring much less data in an optimum way.

- SAR tomography (TomoSAR) [9]-[13] : TomoSAR uses stacks of repeat-pass SAR acquisitions to reconstruct the reflectivity of the scattering objects along elevation for every azimuth-range pixel. For certain imaging geometries, e.g. in urban environment, the signal is sparse in elevation and there are typically only $0 \sim 4$ scatterers inside an azimuth-range pixel.

- Ground Moving Target Identification (GMTI) [14]: Signatures of moving targets in radar are in fact chirp signals with different Doppler and chirp rates depending on the velocities of the targets. Compared with the stationary background, they are sparse signals.

- Inverse SAR (ISAR) [15], [16]: An ISAR system illuminates a maneuvering target and collects a number of pulses coherently. The image of the interesting - mostly military - target is generally constructed by limited strong scattering centers, representing strong spatial sparsity.

Further applications in radars [17], [18] include, e.g. multiple-input/multiple-output (MIMO) radar [19], through-the-wall radar [20] and ground penetrating radar [21].

- Pan-sharpening and hyperspectral image enhancement [22]-[28]: The goal of both pansharpening and hyperspectral image enhancement is to fuse two images which have high spatial and high spectral resolution, respectively. Sufficiently small images patches normally have a sparse representation in overcomplete dictionaries trained from the data.

- Spectral unmixing for hyperspectral data [29][31]: The goal of spectral unmixing is to identify the materials inside a hyperspectral image pixel. Typically there are only few material classes 
(endmembers) inside a pixel compared to the prodigious endmember spectral library.

- Dimension reduction of hyperspectral imagery: Hyperspectral data are characterized by very rich spectral information, which makes them apt to detecting targets of interest, but also introduce drawbacks caused by their high dimensionality. A high dimensional hyperspectral data cube can be decomposed into a low-rank matrix corrupted by a sparse error matrix [32].

Further applications in optical remote sensing include, e.g. classification [33], target detection [34]-[36], anomaly detection and hyperspectral compressive sensing [37].

- Lidar full waveform analysis [38]: Lidar returns are the convolution of the pulse shape and the reflectivity profile. High range resolution requires deconvolution, which can be regularized by the sparsity assumption.

- 3D water vapor tomography using GNSS and InSAR [39]: As the precipitable water vapor (PWV) deduced from GNSS wet delays and the estimated InSAR wet delays only yield integrated information in 2D, this calls for tomographic approaches to reconstruct 3D water vapor fields which can be sparsely represented in, e.g., a cosine transform basis.

In [40], highlight results from selected applications and overview on sparse reconstruction and compressive sensing in various remote sensing problem will be presented.

\section{FUTURE TRENDS}

After the first harvesting in sparsity exploitation in remote sensing community, further developments mainly lie in answering the following questions:

- Instead of deriving the limits of sparse remote sensing by giving SNR, $\mathrm{N}$ and mathematic conditions, can we change the perspective, i.e., starting from the practical scenarios and user specifications?

- How do intelligent compressive remote sensing systems look like that guarantee high probabilities of precise signal recovery?
- For practical problems, how to decide for conventional methods or CS based algorithms that bring the superior performance but relatively high computational cost?

- Where are further sparse signals or smart sparse signal representations to be exploited in remote sensing and Earth observation?

\section{REFERENCES}

[1] D. L. Donoho, "Compressed sensing," IEEE Trans. Inf. Theory, vol. vol.52, no. 4, pp. pp.1289-1306, 2006.

[2] H. Xu, C. Caramanis, and S. Sanghavi, "Robust PCA via Outlier Pursuit," in Advances in Neural Information Processing Systems 23, J. D. Lafferty, C. K. I. Williams, J. Shawe-Taylor, R. S. Zemel, and A. Culotta, Eds. Curran Associates, Inc., 2010, pp. 2496-2504.

[3] D. Baron, M. F. Duarte, M. B. Wakin, S. Sarvotham, and R. G. Baraniuk, "Distributed Compressive Sensing," ArXiv09013403 Cs Math, Jan. 2009.

[4] K. R. Varshney, M. Cetin, J. W. Fisher, and A. S. Willsky, "Sparse Representation in Structured Dictionaries With Application to Synthetic Aperture Radar," IEEE Trans. Signal Process., vol. 56, no. 8, pp. 3548-3561, Aug. 2008.

[5] L. C. Potter, E. Ertin, J. T. Parker, and M. Cetin, "Sparsity and Compressed Sensing in Radar Imaging," Proc. IEEE, vol. vol.98, No.6, pp. 1006-1020, 2010.

[6] M. Tello Alonso, P. Lopez-Dekker, and J. J. Mallorqui, "A Novel Strategy for Radar Imaging Based on Compressive Sensing," IEEE Trans. Geosci. Remote Sens., vol. 48, no. 12, pp. 4285-4295, Dec. 2010.

[7] X. X. Zhu, X.-M. Li, and R. Guo, "Compressive Sensing for Super-resolving SAR Imaging to Support Target Detection in Coastal Zone," 2014, pp. 1-4.

[8] B. Zhang, W. Hong, and Y. Wu, "Sparse microwave imaging: Principles and applications," Sci. China Inf. Sci., vol. 55, no. 8, pp. 1722-1754, Aug. 2012.

[9] X. Zhu and R. Bamler, "Tomographic SAR Inversion by L1-Norm Regularization -- The Compressive Sensing Approach," IEEE Trans. Geosci. Remote Sens., vol. 48, no. 10 , pp. 3839-3846, 2010.

[10] A. Budillon, A. Evangelista, and G. Schirinzi, "ThreeDimensional SAR Focusing From Multipass Signals Using Compressive Sampling," IEEE Trans. Geosci. Remote Sens., vol. 49, no. 1, pp. 488-499, 2011.

[11] E. Aguilera, M. Nannini, and A. Reigber, "A DataAdaptive Compressed Sensing Approach to Polarimetric SAR Tomography of Forested Areas," IEEE Geosci. Remote Sens. Lett., vol. 10, no. 3, pp. 543-547, May 2013.

[12] M. Schmitt and U. Stilla, "Compressive Sensing Based Layover Separation in Airborne Single-Pass MultiBaseline InSAR Data," IEEE Geosci. Remote Sens. Lett., vol. 10, no. 2, pp. 313-317, Mar. 2013.

[13] X. X. Zhu and R. Bamler, "Superresolving SAR Tomography for Multidimensional Imaging of Urban 
Areas: Compressive sensing-based TomoSAR inversion," Signal Process. Mag. IEEE, vol. 31, no. 4, pp. 51-58, Jul. 2014.

[14] L. Pruente, "Application of Compressed Sensing to SAR/GMTI-Data," in 2010 8th European Conference on Synthetic Aperture Radar (EUSAR), 2010, pp. 1-4.

[15] B. Z., Xing M., Qiu C.W., Li J., Sheng J., Li Y. Zhang L., "Resolution enhancement for inversed synthetic aperture radar imaging under low SNR via improved compressive sensing," Trans. Geosci. Remote Sens., vol. 48, no. 10, pp. 3824-3838, Oct. 2010.

[16] H. Liu, B. Jiu, H. Liu, and Z. Bao, "Superresolution ISAR Imaging Based on Sparse Bayesian Learning," IEEE Trans. Geosci. Remote Sens., vol. 52, no. 8, pp. 5005-5013, Aug. 2014.

[17] J. H. G. Ender, "On compressive sensing applied to radar," Signal Process. Spec. Sect. Stat. Signal Array Process., vol. 90, pp. 1402-1414, 2009.

[18] M. Çetin, I. Stojanović, N. O. Önhon, K. R. Varshney, S. Samadi, W. C. Karl, and A. S. Willsky, "Sparsity-Driven Synthetic Aperture Radar Imaging: Reconstruction, autofocusing, moving targets, and compressed sensing," IEEE Signal Process. Mag., vol. 31, no. 4, pp. 27-40, Jul. 2014.

[19] Y. Yu, A. P. Petropulu, and H. V. Poor, "MIMO Radar Using Compressive Sampling," IEEE J. Sel. Top. Signal Process., vol. 4, no. 1, pp. 146-163, Feb. 2010.

[20] Y.-S. Yoon and M. G. Amin, "Compressed sensing technique for high-resolution radar imaging," 2008, vol. 6968, p. 69681A-69681A-10.

[21] A. C. Gurbuz, J. H. McClellan, and W. R. Scott, "A Compressive Sensing Data Acquisition and Imaging Method for Stepped Frequency GPRs," IEEE Trans. Signal Process., vol. 57, no. 7, pp. 2640-2650, Jul. 2009.

[22] Y. B. Li S., "A New Pan-Sharpening Method Using a Compressed Sensing Technique," Trans. Geosci. Remote Sens., vol. 49, no. 2, pp. 738-746, Feb. 2011.

[23] X. X. Zhu and R. Bamler, "A Sparse Image Fusion Algorithm With Application to Pan-Sharpening," IEEE Trans. Geosci. Remote Sens., vol. 51, no. 5, pp. 28272836, May 2013.

[24] C. Jiang, H. Zhang, H. Shen, and L. Zhang, "Two-Step Sparse Coding for the Pan-Sharpening of Remote Sensing Images," IEEE J. Sel. Top. Appl. Earth Obs. Remote Sens., vol. 7, no. 5, pp. 1792-1805, May 2014.

[25] X. X. Zhu, C. Grohnfeldt, and R. Bamler, "Exploiting Joint Sparsity for Pan-sharpening - the J-SparseFI Algorithm," IEEE Trans. Geosci. Remote Sens., 2014.

[26] C. Grohnfeldt, X. X. Zhu, and R. Bamler, "Jointly Sparse Fusion of Hyperspectral and Multispectral Imagery," presented at the Geoscience and Remote Sensing Symposium (IGARSS), 2013 IEEE International, 2013, pp. 4090-4093.

[27] Q. Wei, J. Bioucas-Dias, N. Dobigeon, and J.-Y. Tourneret, "Hyperspectral and Multispectral Image Fusion Based on a Sparse Representation," IEEE Trans.
Geosci. Remote Sens., vol. 53, no. 7, pp. 3658-3668, Jul 2015.

[28] C. Grohnfeldt, S. Peter, and X. X. Zhu, "Jointly Sparse Fusion of Hyperspectral and Multispectral Imagery -The J-SparseFI-HM Algorithm," IEEE Trans. Geosci. Remote Sens., no. submitted.

[29] Z. Guo, T. Wittman, and S. Osher, "L1 unmixing and its application to hyperspectral image enhancement," in Proceedings SPIE Conference on Algorithms and Technologies for Multispectral, Hyperspectral, and Ultraspectral Imagery XV, 2009, vol. 7334, p. 73341M$73341 \mathrm{M}$.

[30] M.-D. Iordache, J. M. Bioucas-Dias, and A. Plaza, "Sparse Unmixing of Hyperspectral Data," Geosci. Remote Sens. IEEE Trans. On, vol. 49, no. 6, pp. 2014 2039, 2011.

[31] J. Bieniarz, E. Aguilera, X. X. Zhu, R. Muller, and P. Reinartz, "Joint Sparsity Model for Multilook Hyperspectral Image Unmixing," IEEE Geosci. Remote Sens. Lett., vol. PP, no. 99, pp. 1-5, 2014.

[32] Q. Du and J. E. Fowler, "Low-Complexity Principal Component Analysis for Hyperspectral Image Compression," Int. J. High Perform. Comput. Appl., vol. 22, no. 4, pp. 438-448, Nov. 2008.

[33] X. Sun, N. M. Nasrabadi, and T. D. Tran, “Task-Driven Dictionary Learning for Hyperspectral Image Classification With Structured Sparsity Constraints," IEEE Trans. Geosci. Remote Sens., vol. 53, no. 8, pp. 4457-4471, Aug. 2015.

[34] Y. Chen, N. M. Nasrabadi, and T. D. Tran, "Sparse Representation for Target Detection in Hyperspectral Imagery," IEEE J. Sel. Top. Signal Process., vol. 5, no. 3, pp. 629-640, Jun. 2011.

[35] N. Yokoya and A. Iwasaki, "Object Detection Based on Sparse Representation and Hough Voting for Optical Remote Sensing Imagery," IEEE J. Sel. Top. Appl. Earth Obs. Remote Sens., vol. PP, no. 99, pp. 1-1, 2015.

[36] Y. Zhang, B. Du, and L. Zhang, "A Sparse Representation-Based Binary Hypothesis Model for Target Detection in Hyperspectral Images," IEEE Trans. Geosci. Remote Sens., vol. 53, no. 3, pp. 1346-1354, Mar. 2015.

[37] G. Martin, J. M. Bioucas-Dias, and A. Plaza, "HYCA: A New Technique for Hyperspectral Compressive Sensing," IEEE Trans. Geosci. Remote Sens., vol. 53, no. 5, pp. 2819-2831, May 2015.

[38] T. C., Zaletnyik P. Laky S., "Compressing and classifying LiDAR waveform data," Proceeding Int. LiDAR Mapp. Forum, 2010.

[39] M. Heublein, X. X. Zhu, F. Alshawaf, M. Mayer, R. Bamler, and S. Hinz, "Compressive Sensing for Neutrospheric Water Vapor Tomography from GNSS and InSAR Observations," presented at the Geoscience and Remote Sensing Symposium (IGARSS), 2015 IEEE International, Milan, Italy, submitted, 2015.

[40] X. X. Zhu and R. Bamler, Sparse Reconstruction and Compressive Sensing in Remote Sensing, In editing, expected to be published in March 2016. Springer, 2016. 